\title{
Inadequacies in the conventional treatment of the radiation field of moving sources
}

\author{
Houshang Ardavan \\ Institute of Astronomy, University of Cambridge, \\ Madingley Road, Cambridge CB3 OHA, UK \\ Arzhang Ardavan \\ Clarendon Laboratory, Department of Physics, \\ University of Oxford, Parks Road, Oxford OX1 3PU, UK \\ John Singleton \\ National High Magnetic Field Laboratory, MS-E536, \\ Los Alamos National Laboratory, Los Alamos, New Mexico 87545 \\ Joseph Fasel and Andrea Schmidt \\ Process Modeling and Analysis, MS-E548, \\ Los Alamos National Laboratory, Los Alamos, New Mexico 87545
}

(Dated: 2009 May 28) 


\begin{abstract}
There is a fundamental difference between the classical expression for the retarded electromagnetic potential and the corresponding retarded solution of the wave equation that governs the electromagnetic field. While the boundary contribution to the retarded solution for the potential can always be rendered equal to zero by means of a gauge transformation that preserves the Lorenz condition, the boundary contribution to the retarded solution of the wave equation governing the field may be neglected only if it diminishes with distance faster than the contribution of the source density in the far zone. In the case of a source whose distribution pattern both rotates and travels faster than light in vacuo, as realized in recent experiments, the boundary term in the retarded solution governing the field is by a factor of the order of $R^{1 / 2}$ larger than the source term of this solution in the limit that the distance $R$ of the boundary from the source tends to infinity. This result is consistent with the prediction of the retarded potential that part of the radiation field generated by a rotating superluminal source decays as $R^{-1 / 2}$, instead of $R^{-1}$, a prediction that is confirmed experimentally. More importantly, it pinpoints the reason why an argument based on a solution of the wave equation governing the field in which the boundary term is neglected (such as appears in the published literature) misses the nonspherical decay of the field.
\end{abstract}




\section{INTRODUCTION}

Scientific investigation of the electromagnetic field generated by a charged particle that moves faster than light began with a largely ignored article by physicist and mathematician Oliver Heaviside in $1887^{1}$ and is the subject of several papers by Sommerfeld in 1904 and early 1905! ${ }^{2}$ The publication of the special theory of relativity in June $1905, \frac{3}{2}$ however, discouraged further work as one of its tenets is, of course, that any known particle that has a charge also has a rest mass and so is barred from moving faster than light. Moreover, no source that moves faster than the wave speed can be pointlike, for this results in infinitely strong fields on the envelope of the emitted wave fronts. It was not until the early 1970s that Bolotovskii and Ginzburg $4 \sqrt{45}$ pointed out that although special relativity precludes massive particles from moving superluminally, patterns of distribution of extended sources can move faster than light as a result of the coordinated motion of their constituent charged particles, which

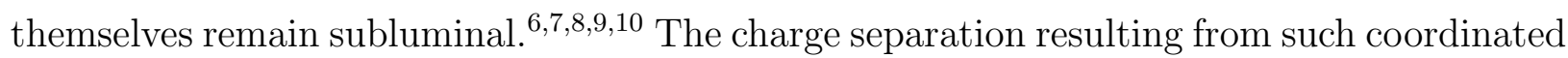
motion gives rise to a polarization current whose distribution pattern can move faster than light in vacuo. The way in which this current acts as a source of radiation is immediately apparent from the Ampère-Maxwell equation (SI units),

$$
\boldsymbol{\nabla} \times \mathbf{H}=\mathbf{J}+\frac{\partial \mathbf{D}}{\partial t}=\mathbf{J}+\epsilon_{0} \frac{\partial \mathbf{E}}{\partial t}+\frac{\partial \mathbf{P}}{\partial t},
$$

in which we see that the polarization current $\partial \mathbf{P} / \partial t$ contributes to the magnetic field $\mathbf{H}$ in just the same way as the current $\mathbf{J}$ of free charges. However, as $\partial \mathbf{P} / \partial t$ is not carried by massive particles, it is not limited to subluminal speeds. We should clarify that while the source distribution is superluminal, the emitted radiation (as any other) travels at the speed of light.

Extended sources of electromagnetic radiation whose distribution patterns move faster than light in vacuo have been experimentally realized by several groups ${ }^{6 / 711 / 12}$ and have many potential applications in science and technology $\frac{13}{13}$ Furthermore, they may be responsible for the extreme properties of the electromagnetic radiation received from astronomical objects such as pulsars (rapidly spinning, highly magnetized neutron stars) $\frac{1014 \mid 15}{15}$ Methods of dealing with the asymptotic behavior of the radiation emitted by such sources, however, are largely neglected in standard texts ${ }^{16}$ More dangerously, certain textbook formulæ, derived in the context of stationary or subluminally moving sources, cannot be used in treating unusual cases such as those in which the radiation remains focused in the far zone. ${ }^{819[17} \mathrm{An}$ 
example is this expression for the far-zone magnetic field of a localized current with the density $\mathbf{j}$ :

$$
\mathbf{B}\left(\mathbf{x}_{\mathrm{P}}, t_{\mathrm{P}}\right) \simeq \frac{1}{c} \int \mathrm{d}^{3} x \frac{[\boldsymbol{\nabla} \times \mathbf{j}]}{\left|\mathbf{x}_{\mathrm{P}}-\mathbf{x}\right|} .
$$

Here, $\left(\mathbf{x}_{\mathrm{P}}, t_{\mathrm{P}}\right)$ and $(\mathbf{x}, t)$ are the space-time coordinates of the observation point and the source points, respectively, and the square brackets denote the retarded value of $\boldsymbol{\nabla} \times \mathbf{j}$. (Note that, in contrast to the free-charge current $\mathbf{J}$ of Eq. (1), this $\mathbf{j}$ is a generalized current that also includes $\partial \mathbf{D} / \partial t$.) The derivation of Eq. (2) involves the neglect, in the far zone, of a boundary term containing the gradient of the radiation field. In the case of a conventional source, this term decays more rapidly with distance than the integral that remains in Eq. (2). This is not the case, however, for the radiation field of a polarization current whose distribution pattern has an accelerated motion with a speed exceeding that of light, $c .^{[1718119}$ In the next section, we describe such a source, the emission from which consists of a collection of narrowing subbeams for which the absolute value of the gradient of the radiation field $\mathbf{B}$ increases (as $R_{\mathrm{P}}^{7 / 2}$ ) with the distance $R_{\mathrm{P}}$ from its source.

Since the exact form of Eq. (2) entails an additional boundary term that depends on the gradient of the field in the far zone [Eq. (13), below], the increase in the magnitude of the gradient of the present radiation field with distance renders the boundary term in the retarded solution of Maxwell's equation for B dominant over its source term. The inadequacy of Eq. (2) in describing the radiation field generated by a superluminal source lies in the neglect of this boundary term.

The remainder of this paper is organized as follows. Section II describes the superluminally-rotating source distribution, and a practical experimental realization of such a source. In order to make the later discussion more comprehensible, Section III considers a small volume element of this source and discusses the superposition of multiple retarded times that render the field of a point source divergent. Section IV generalizes this discussion to an extended source and describes the morphology of the resulting radiation "beam", providing a geometrical argument as to why the boundary term missing from Eq. 2 dominates in the far field, and Section V describes supporting experimental data. Sections VI and VII contain the main, substantive point of the current work; while the boundary contribution to the retarded solution for the potential can always be rendered equal to zero by means of a gauge transformation, the boundary contribution to the retarded solution of the wave equation governing the field cannot be neglected in the case of a superluminal source. Finally 
Section VIII gives a brief summary.

\section{A SUPERLUMINALLY ROTATING SOURCE DISTRIBUTION}

Here we consider a particular moving source distribution for which Eq. (2) is not an adequate description of the radiation field in the far zone and its experimental realization. The findings of Refs. [9,17] fully clarify how this case should be treated in order to obtain results consistent with experimental data. In these papers, we solved the inhomogeneous wave equation governing the electromagnetic potential [Eq. (7) below] in unbounded space, under null initial conditions, with a polarization current density $\mathbf{j}=\partial \mathbf{P} / \partial t$ for which

$$
P_{r, \varphi, z}(r, \varphi, z, t)=s_{r, \varphi, z}(r, z) \cos (m \hat{\varphi}) \cos (\Omega t), \quad-\pi<\hat{\varphi} \leq \pi
$$

where

$$
\hat{\varphi} \equiv \varphi-\omega t
$$

Here, $P_{r, \varphi, z}$ are the components of the polarization $\mathbf{P}$ in a cylindrical coordinate system based on the axis of rotation, $\omega$ and $\Omega$ are angular frequencies, $\mathbf{s}(r, z)$ is an arbitrary vector function with a finite support in $r>c / \omega$, and $m$ is a positive integer. For fixed $t$, the azimuthal dependence of the polarization (3) along each circle of radius $r$ within the source is the same as that of a sinusoidal wave train, of wavelength $2 \pi r / m$, whose $m$ cycles fit around the circumference of the circle smoothly. As time elapses, this wave train both propagates around each circle with the superluminal velocity $r \omega$ and oscillates in its amplitude with frequency $\Omega$. This is a generic source: one can construct any distribution with a uniformly rotating pattern, $P_{r, \varphi, z}(r, \hat{\varphi}, z)$, by the superposition over $m$ of terms of the form $s_{r, \varphi, z}(r, z, m) \cos (m \hat{\varphi})$. For example, the polarization-current patterns responsible for the pulsar radiation visible from Earth are thought to be animated by the superluminal rotation of the neutron-star core's intense magnetic field through the pulsar's plasma atmosphere ${ }^{14}$; one can envisage representing this rotating pattern by summing such terms. Moreover, Eq. (3) corresponds

to a laboratory-based source that has been used in experimental demonstrations of some of the phenomena described below ${ }^{6 / 7}$

The experimental apparatus in question (see Fig. 1) consists of a continuous strip of dielectric material on top of which is placed an array of metal electrodes; underneath is a continuous ground plate. Each upper electrode is connected to an individual amplifier, so 
that by turning the amplifiers on and off in sequence one can apply voltages that generate a polarized region that moves along the dielectric with an arbitrarily high speed. In practice, the dielectric is a strip of alumina $10 \mathrm{~mm}$ thick and $50 \mathrm{~mm}$ across, corresponding to a $10^{\circ}$ arc of a circle of average radius $10.025 \mathrm{~m}$. Above the alumina strip, there are 41 upper electrodes of mean width $42.6 \mathrm{~mm}$, with centers $44.6 \mathrm{~mm}$ apart ${ }^{617}$

The voltage $V_{j}$ applied to the $j$ th electrode is of the form

$$
V_{j}=V_{0} \cos [m \omega(j \Delta t-t)] \cos (\Omega t),
$$

where $m \omega$ and $\Omega$ are as defined in Eq. (3): the first cosine gives rise to the polarizationcurrent wave that propagates along the dielectric and the second to a modulation of this wave. The source speed $v$ is determined by the phase difference between the oscillations of neighboring electrodes; given the dimensions of the electrodes of the experimental machine, a superluminal speed $v \geq c$ is obtained for $\Delta t \leq 148.8 \mathrm{ps}$. In the experiments reported in Refs. 66.7], $m \omega /(2 \pi)$ and $\Omega /(2 \pi)$ had the values $552.645 \mathrm{MHz}$ and $46.042 \mathrm{MHz}$, respectively, and the source was run at $v / c=0.875,1.064,1.25$ and 2.00 (see Refs. [6]7] for a more complete description).

\section{THE FIELD GENERATED BY A CONSTITUENT VOLUME ELEMENT OF THE SOURCE}

A superluminal source is necessarily volume-distributed ${ }^{4}$ However, its field can be built up from the superposition of the fields of its small, moving constituent volume elements. Figure 2(a) shows that the waves generated by such a volume element of a rotating superluminal source possess a cusped envelope and that, inside the envelope, three wave fronts pass through any given observation point simultaneously. This reflects the fact that the field inside the envelope receives simultaneous contributions from three distinct values of the retarded time [see Fig. 2(c)]. On the cusp of the envelope, where the space-time trajectory of the source is tangent to the past light cone of the observer [Fig. 2(d)], all three contributions toward the value of the field coalesce. $\frac{821222}{2}$

On this cusp (caustic), the source approaches the observer with the speed of light and zero acceleration at the retarded time, i.e. $\mathrm{d} R(t) / \mathrm{d} t=-c$ and $\mathrm{d}^{2} R(t) / \mathrm{d} t^{2}=0$, where $R(t) \equiv\left|\mathbf{x}(t)-\mathbf{x}_{\mathbf{P}}\right|$ is the distance between the source point $\mathbf{x}(t)$ and the observation point 
(a)

$\ominus \ominus \ominus \ominus \ominus \ominus \ominus \ominus \ominus \ominus \ominus \ominus \ominus \ominus \ominus \ominus \oplus$

(b)

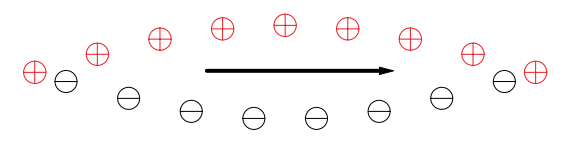

(c)

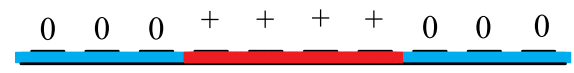

(d)

$\underline{0} \quad 0 \quad 0 \quad 0 \quad+\quad+\quad+\quad+\quad 0 \quad 0$

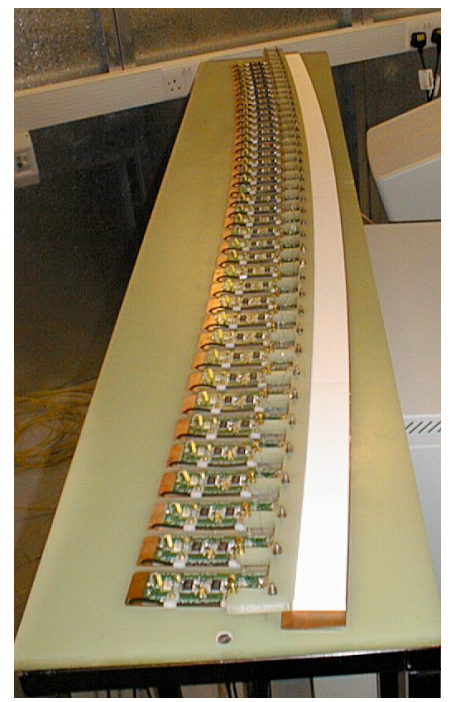

(e)

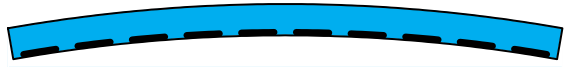

FIG. 1: Left: experimental animation of a superluminal polarization current. (a) A simplified dielectric solid containing negative $(\ominus)$ and positive $(\oplus)$ ions. In (b), a spatially-varying electric field has been applied, causing the positive and negative ions to move in opposite directions; a finite polarization $\mathbf{P}$ has therefore been induced. If the spatially-varying field is made to move along the direction of the arrow, the polarized region moves with it. (c) Schematic side view of a practical superluminal source, showing metal electrodes above a strip of dielectric (shaded region) and a ground plate below it. "0" indicates that there is no voltage on that particular upper electrode; the symbol + indicates a positive voltage applied to the upper electrode. The voltage on the electrodes produces a finite polarization of the dielectric (darker shading). (d) By switching the voltages on the electrodes on and off, the polarized region (darker shading) can be made to move along the dielectric. (e) Top view, showing the curvature of the dielectric (lighter shaded region). The curvature introduces centripetal acceleration in the moving polarized region. Note that the electrodes (black shading) cover only part of the top surface of the dielectric. Right: a photograph of the experimental apparatus showing a curved strip of dielectric material (alumina) sandwiched between a copper ground plate (below) and 41 electrodes, each connected to its own amplifier (left).

$\mathbf{x}_{\mathrm{P}}$. As a result, the interval of emission time for the signal carried by the cusp is much longer than the interval of its reception time. ${ }^{22}$ Fig. 2 (d) represents this diagrammatically; rather than merely intersecting at one or three points, the space-time trajectory of the source and the observer's light cone become tangent to one another. This essentially instantaneous 


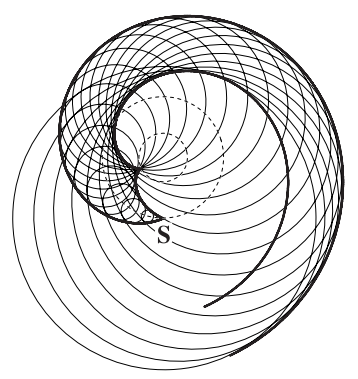

(a)

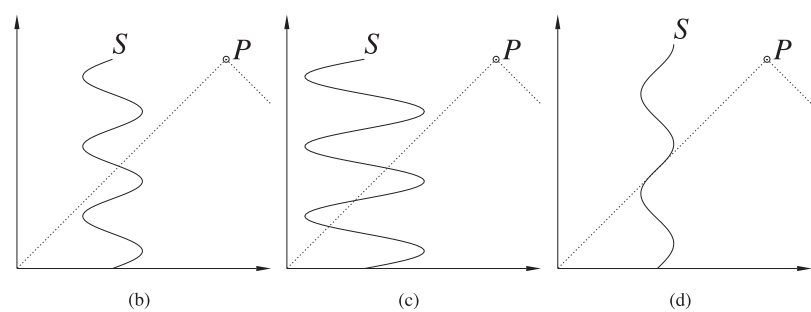

FIG. 2: (a) Envelope of the spherical wave fronts emanating from a superluminally moving source element $(S)$ in uniform circular motion. The lighter circles are Huygen's wavelets emitted by the source as it traverses its circular orbit, designated by the larger of the two dotted circles. The heavy curves show the cross section of the envelope of the wavelets within the plane of the orbit of the source. The smaller of the dotted circles represents the light cylinder $r=c / \omega$. (b), (c) and (d) Space-time (i.e. ct versus distance $x$ ) diagrams showing the intersection of the trajectory of the source point $\mathrm{S}$ with the past light cone of the observation point $\mathrm{P}$ when $\mathrm{P}$ lies outside (b), inside (c), and on the cusp of (d) the envelope of wave fronts.

reception of contributions from an extended period of emission time represents focusing of the radiation in the time domain; it has been described as temporal focusing and is the subject of two experimental papers. $\frac{617}{}$ It is this temporal focusing that leads to the divergence of the field of a point source on the cusp of the envelope of wave fronts and is ultimately responsible for the component of the radiation from an extended source whose intensity decays as 1 /distance, rather than the inverse square law that applies to all other sources 9117 ; consequently, this part of the radiation will dominate in observations made from large distances.

A three-dimensional view of the envelope of wave fronts and its cusp is shown in Fig. 3 . The two sheets of the envelope, and the cusp along which these two sheets meet tangentially, spiral outward into the far zone. In the far zone, the cusp lies on the double cone 


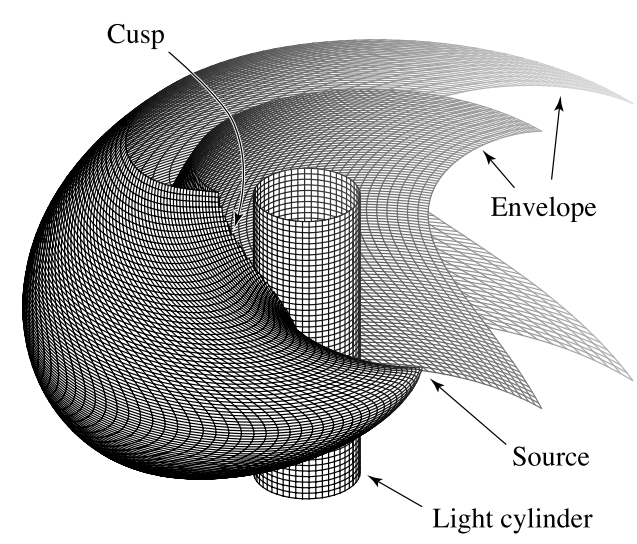

(a)

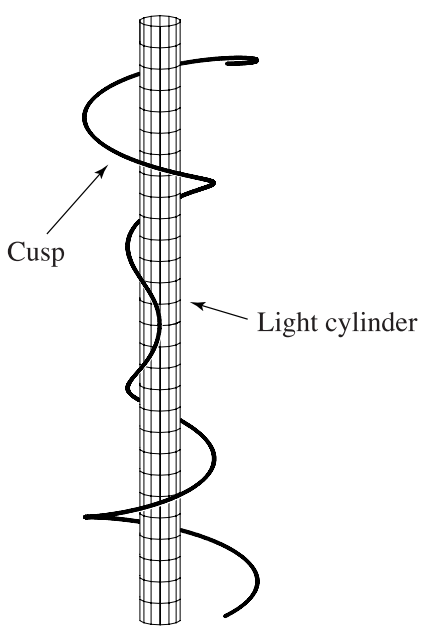

(b)

FIG. 3: Three-dimensional views of the envelope (a) and its cusp (b).

$\theta_{\mathrm{P}}=\arcsin [c /(r \omega)], \theta_{\mathrm{P}}=\pi-\arcsin [c /(r \omega)]$, where $\left(R_{\mathrm{P}}, \theta_{\mathrm{P}}, \varphi_{\mathrm{P}}\right)$ denote the spherical polar coordinates of the observation point P. Thus, a stationary observer in the polar interval $\arcsin [c /(r \omega)] \leq \theta_{\mathrm{P}} \leq \pi-\arcsin [c /(r \omega)]$ receives recurring pulses from each volume element as the envelope rotates past him/her ${ }^{9}$

Figure 4 shows the radiation field generated by the rotating source element $S$ on a cone close to the cusp, just outside the envelope. Not only does the spiraling cusp embody a recurring pulse, but the plane of polarization of the radiation swings across the pulse, $\frac{10}{10}$ as does the radio emission received from pulsars. 23

\section{THE FIELD GENERATED BY THE ENTIRE VOLUME OF THE SOURCE}

The dominant contribution towards the field of an extended source comes from a thin filamentary part of the source that approaches the observer, along the radiation direction, with the speed of light and zero acceleration at the retarded time ${ }^{9}$ For an observation point $\mathrm{P}$ in the far zone with the coordinates $\left(R_{\mathrm{P}}, \theta_{\mathrm{P}}, \varphi_{\mathrm{P}}\right)$, this filament is located at $r=(c / \omega) \csc \theta_{\mathrm{P}}$, $\varphi=\varphi_{\mathrm{P}}+3 \pi / 2$ and is essentially parallel to the rotation axis (Fig. 5). The collection

of cusps of the envelopes of wave fronts that emanate from various volume elements of the contributing filament form a subbeam whose polar width is nondiffracting: the linear dimension of this bundle of cusps in the direction parallel to the rotation axis remains the same at all distances from the source, so that the polar angle $\delta \theta_{\mathrm{P}}$ subtended by the subbeam 


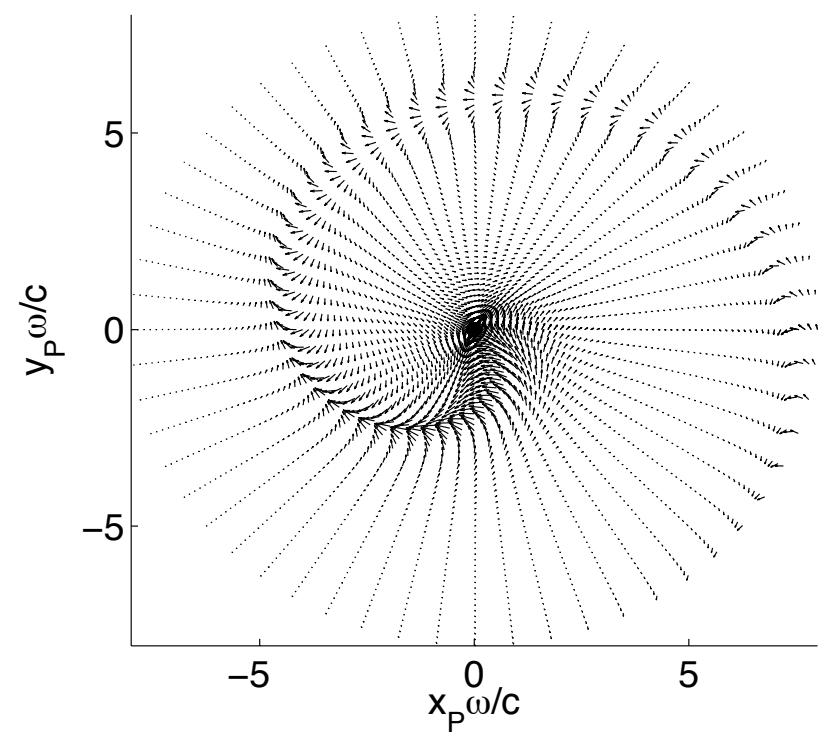

FIG. 4: Polarization position angles and field strengths on the cone $\theta_{\mathrm{P}}=\pi / 12$ outside the envelope of wave fronts for a source element with $r \omega=2$. Here, the field vectors are projected onto the plane $\left(x_{\mathrm{P}}, y_{\mathrm{P}}\right)$ of the source's orbit.

decreases as $R_{\mathrm{P}}^{-1}$ with increasing $R_{\mathrm{P}}$ (Fig. 5 and Ref. [9]).

In that it consists of caustics and so is constantly dispersed and reconstructed out of other waves, the subbeam in question radically differs from a conventional radiation beam (see Appendix D of Ref. [21]). The narrowing of its polar width (as $R_{\mathrm{P}}{ }^{-1}$ ) is accompanied by a more slowly diminishing intensity (an intensity that diminishes as $R_{\mathrm{P}}^{-1}$ instead of $R_{\mathrm{P}}{ }^{-2}$ with distance), so that the flux of energy across its cross sectional area remains the same for all $R_{\mathrm{P}} \stackrel{9}{9}$ As we will see in the next section, this slower rate of decay of the emission from a superluminally rotating source has been confirmed experimentally upto several hundred Fresnel distances (Rayleigh ranges)!

The contributing part of an extended source (the filament that approaches the observation point with the speed of light and zero acceleration) changes as the source rotates (see Fig. 5). In the case of a turbulent plasma with a superluminally rotating macroscopic distribution, therefore, the overall beam within which the narrow, nonspherically decaying radiation is detectable would consist of an incoherent superposition of coherent, nondiffracting subbeams with widely differing amplitudes and phases (similar to the train of giant pulses received from the Crab pulsar)!24 


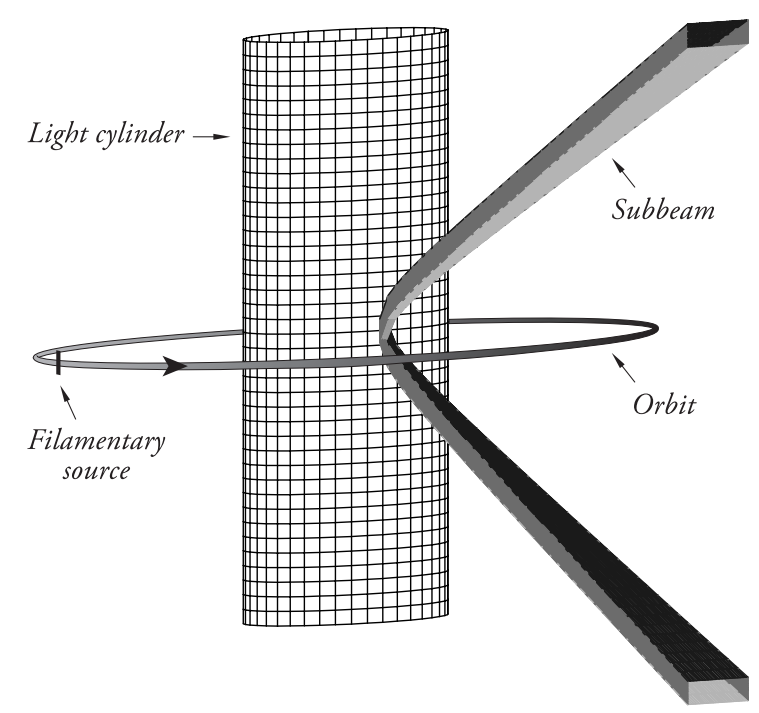

FIG. 5: Schematic illustration of the light cylinder $r=c / \omega$, the filamentary part of the source that approaches the observation point with the speed of light and zero acceleration at the retarded time, the orbit $r=c /\left(\omega \sin \theta_{\mathrm{P}}\right)$ of this filamentary source, and the subbeam formed by the bundle of cusps that emanate from the constituent volume elements of this filament.

The overall beam occupies a solid angle whose polar and azimuthal extents are independent of the distance $R_{\mathrm{P}}$. It is detectable within the polar interval $\arccos \left[\left(r_{l} \omega / c\right)^{-1}\right] \leq$ $\left|\theta_{\mathrm{P}}-\pi / 2\right| \leq \arccos \left[\left(r_{u} \omega / c\right)^{-1}\right]$, where $\left[r_{l}, r_{u}\right]$ denotes the radial extent of the superluminal part of the source. The azimuthal profile of this overall beam reflects the distribution of the source density around the cylinder $r=c /\left(\omega \sin \theta_{\mathrm{P}}\right)$, from which the dominant contribution to the radiation arises $\frac{9}{9}$

Because the subbeams that constitute the overall beam are narrower the farther away from the source they are detected, the absolute value of the gradient of the radiation field associated with them increases with distance: it has been explicitly shown in Ref. [17] that the gradient of the nonspherically decaying field generated by the superluminal source described in Eq. (3) has a magnitude that increases as $R_{\mathrm{P}}{ }^{7 / 2}$ with increasing $R_{\mathrm{P}}$.

\section{EXPERIMENTAL AND OBSERVATIONAL CONFIRMATION THAT THE NONSPHERICALLY-DECAYING TERM DOMINATES IN THE FAR FIELD}

The issue under discussion in this paper is the dominance of the boundary term neglected in Eq. (2) in the far field. Experimental demonstrations of the far-field dominance of the 


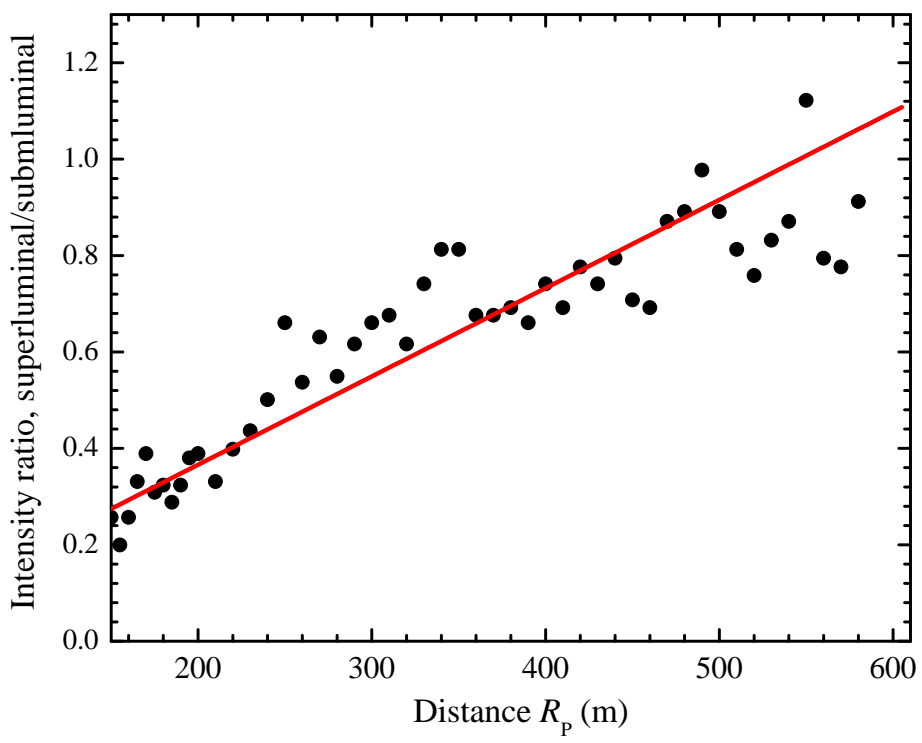

FIG. 6: (a) and (b): Ratio of the detected intensity with the experimental source (Fig. 1) running superluminally (at $v / c=1.064)$ to that with the source running subluminally $(v / c=0.875)$ versus distance $R_{\mathrm{P}}$. For both data sets the detector was moved along a path close to the direction of the cusp expected for $v / c=1.064$. Data are points; the line is a straight line through the origin, which implies that the intensity in the superluminal case would decline in free space as $1 / R_{\mathrm{P}}$ (after Ref. (6)).

boundary term focus on detecting emissions whose intensity decays with distance as $1 / R_{\mathrm{P}}$, rather that the more conventional inverse-square law.

Fig. 6 shows a ground-based demonstration ${ }^{6}$ using the machine depicted in Fig. 1. The experiment consisted of moving a detector along the expected cusp direction for a source speed of $v / c=1.064$ and recording the intensity as a function of distance. The source speed was then set to a subluminal velocity (i.e. no cusp formation- intensity follows the inversesquare law with distance) and the experiment repeated with the detector following the same path. The ratio of the detected intensity for $v / c=1.064$ to that for the machine running at subluminal speed increases linearly with distance, showing that the cusp radiation intensity is decaying more slowly with increasing distance, in line with expectations $[$ [

For demonstrations covering much larger distances, one must turn to astronomical observations. In a recent paper, ${ }^{33}$ the Maximum Likelihood Method ${ }^{34}$ has been applied to 

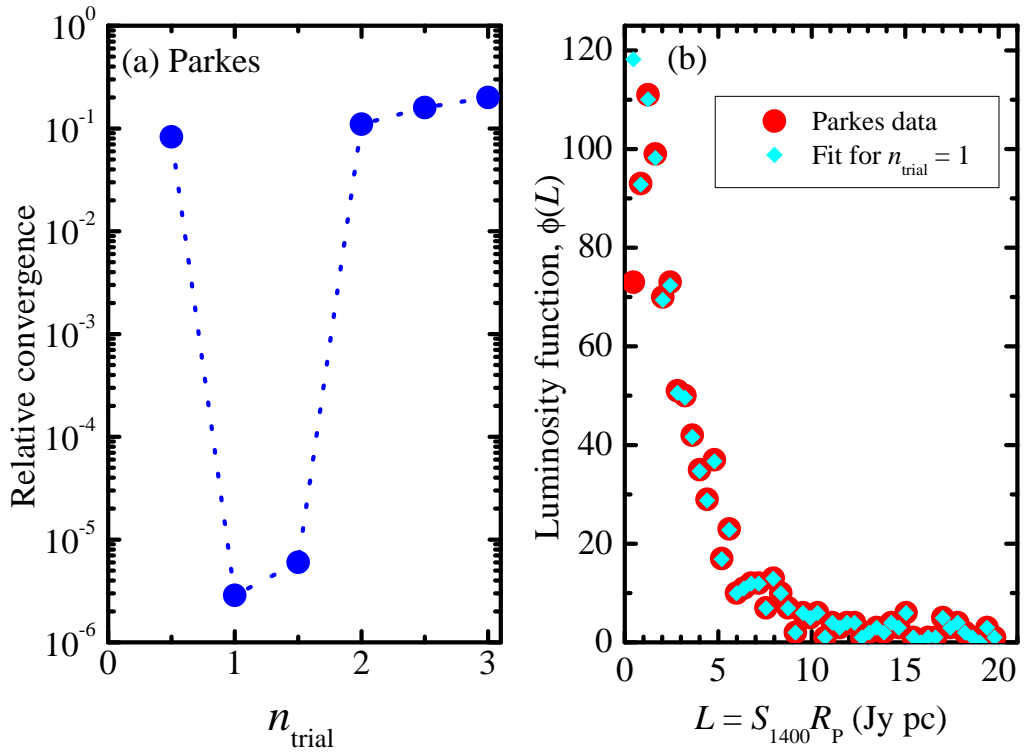

FIG. 7: Demonstration that the far-field emission of pulsars is dominated by a component that decays nonspherically with distance (after Ref. (33)). (a) The relative convergence error from the Maximum Likelihood Method applied to 971 intragalactic pulsars (from the Parkes Multibeam Survey) as a function of the trial exponent $n_{\text {trial }}$. There is a clear minimum at $n_{\text {trial }}=1$, strongly suggesting that the flux at $1400 \mathrm{MHz}, S_{1400} \propto 1 / R_{\mathrm{P}}$. (b) The inferred luminosity function with raw pulsar data (taken from the Parkes Multibeam Survey) plotted as large circles, and the fitted luminosity function as small diamonds. It is clear that there is a very close correspondence between fit and data.

observational data from 971 pulsars to deduce the likely power law with which their flux $S$ (a quantity proportional to intensity) falls off with distance. The Maximum Likelihood Method determines the probable luminosity function (i.e. the distribution of pulsars with respect to their radiated power) whilst making allowances for missing observations due to the low sensitivity of Earth-bound instruments. 33 The statistical success of the method is measured using a relative convergence error; to find the exponent $n$ in the relationship $S \propto 1 / R_{\mathrm{P}}^{n}$, the calculation is repeated for several different values of a trial exponent, $n_{\text {trial }}$ until the value that gives the smallest relative convergence error is located. As can be seen in Fig. 7, the pulsar data are most convincingly fitted by a flux $S \propto 1 / R_{\mathrm{P}}$. 33

In summary, experimental and observational data suggest very strongly that the boundary 
term neglected in Eq. (2) dominates in the far field, resulting in a component of the radiation that is nonspherically decaying, with an intensity proportional to $1 / R_{\mathrm{P}}$.

\section{FUNDAMENTAL RÔLE OF THE RETARDED POTENTIAL}

While the results of an analysis based on the retarded solution to the wave equation governing the field depend crucially on the boundary conditions satisfied by the field at infinity, $\frac{17}{17}$ we instead base our analysis on the retarded potential, we require no corresponding explicit knowledge of the value of the potential in the radiation zone. By using the field derived from the retarded potential [Eq. (11)], we will evaluate the boundary term in the retarded solution to the wave equation governing the field [Eq. (13)] and show that, far from being equal to zero, as assumed in Refs. [16,25,26,27,28,29], this boundary term constitutes the dominant contribution to the value of the field in the far zone. There is no discrepancy between the results obtained from the retarded solution for the potential and the retarded solution for the field once the boundary term in the solution to the wave equation governing the field is retained. Furthermore, this analysis is robust with respect to choice of integration boundaries (see the Appendix).

We first describe how the boundary contribution to the retarded solution for the potential can always be made equal to zero, irrespective of the source motion.

In the Lorenz gauge, $\left[\right.$ i.e., the choice of a set of potentials $\left(\mathbf{A}, A^{0}\right)$ that satisfy the Lorenz condition $\left.\boldsymbol{\nabla} \cdot \mathbf{A}+c^{-2} \partial A^{0} / \partial t=0\right]$, the electromagnetic fields

$$
\mathbf{E}=-\nabla_{\mathrm{P}} A^{0}-\partial \mathbf{A} / \partial\left(c t_{\mathrm{P}}\right), \quad \mathbf{B}=\nabla_{\mathrm{P}} \times \mathbf{A}
$$

are given by a four-potential $A^{\mu}$ that satisfies the wave equation

$$
\nabla^{2} A^{\mu}-\frac{1}{c^{2}} \frac{\partial^{2} A^{\mu}}{\partial t^{2}}=-\frac{4 \pi}{c} j^{\mu}, \quad \mu=0, \cdots, 3
$$

where $A^{0} / c$ and $j^{0} / c$ are the electric potential and charge density and $A^{\mu}$ and $j^{\mu}$ for $\mu=1,2,3$

are the Cartesian components of the magnetic potential $\mathbf{A}$ and the current density $\mathbf{j} \cdot \frac{16}{16}$ The solution to the initial-boundary value problem for Eq. (7) is given by

$$
\begin{aligned}
A^{\mu}\left(\mathbf{x}_{\mathrm{P}}, t_{\mathrm{P}}\right)= & \frac{1}{c} \int_{0}^{t_{\mathrm{P}}} \mathrm{d} t \int_{V} \mathrm{~d}^{3} x j^{\mu} G+\frac{1}{4 \pi} \int_{0}^{t_{\mathrm{P}}} \mathrm{d} t \int_{\Sigma} \mathrm{d} \mathbf{S} \cdot\left(G \nabla A^{\mu}-A^{\mu} \nabla G\right) \\
& -\frac{1}{4 \pi c^{2}} \int_{V} \mathrm{~d}^{3} x\left(A^{\mu} \frac{\partial G}{\partial t}-G \frac{\partial A^{\mu}}{\partial t}\right)_{t=0},
\end{aligned}
$$


in which $G$ is the Green's function and $\Sigma$ is the surface enclosing the volume $V$ (see, e.g., page 893 of Ref. [30]).

The potential arising from a general time-dependent localized source in unbounded space decays as $R_{\mathrm{P}}^{-1}$ when $R_{\mathrm{P}} \equiv\left|\mathbf{x}_{\mathrm{P}}\right| \rightarrow \infty$, so that for an arbitrary free-space potential the second term in Eq. (8) would be of the same order of magnitude $\left(\sim R_{\mathrm{P}}^{-1}\right)$ as the first term in the limit that the boundary $\Sigma$ tends to infinity. However, even those potentials that satisfy the Lorenz condition are arbitrary to within a solution of the homogeneous wave equation: the gauge transformation

$$
\mathbf{A} \rightarrow \mathbf{A}+\nabla \Lambda, \quad A^{0} \rightarrow A^{0}-\partial \Lambda / \partial t
$$

preserves the Lorenz condition if $\nabla^{2} \Lambda-c^{-2} \partial^{2} \Lambda / \partial t^{2}=0 ! 12$ One can always use this gauge freedom in the choice of the potential to render the boundary contribution (the second term) in Eq. (8) equal to zero, since this term, too, satisfies the homogeneous wave equation. Under the null initial conditions $\left.A^{\mu}\right|_{t=0}=\left(\partial A^{\mu} / \partial t\right)_{t=0}=0$ assumed in this paper, the contribution from the third term in Eq. (8) is identically zero.

In the absence of boundaries, the retarded Green's function has the form

$$
G\left(\mathbf{x}, t ; \mathbf{x}_{\mathrm{P}}, t_{\mathrm{P}}\right)=\frac{\delta\left(t_{\mathrm{P}}-t-R / c\right)}{R}
$$

where $\delta$ is the Dirac delta function and $R$ is the magnitude of the separation $\mathbf{R} \equiv \mathbf{x}_{\mathrm{P}}-\mathbf{x}$ between the observation point $\mathbf{x}_{\mathrm{P}}$ and the source point $\mathbf{x}$. Irrespective of whether the radiation decays spherically (as in the case of a conventional source) or nonspherically (as applies for a rotating superluminal source - see Section II] ${ }^{917}$ therefore, the potential $A^{\mu}$ due to a localized source distribution that is switched on at $t=0$ in an unbounded space, can be calculated from the first term in Eq. (8):

$$
A^{\mu}\left(\mathbf{x}_{\mathrm{P}}, t_{\mathrm{P}}\right)=c^{-1} \int \mathrm{d}^{3} x \mathrm{~d} t j^{\mu}(\mathbf{x}, t) \delta\left(t_{\mathrm{P}}-t-R / c\right) / R
$$

i.e., from the classical expression for the retarded potential. Whatever the Green's function for the problem may be, in the presence of boundaries, it approaches the expression in Eq. (10) in the limit where the boundaries tend to infinity. Hence one can also use this potential to calculate the field on a boundary that lies at large distances from the source. 


\section{RETARDED SOLUTION OF THE EQUATION GOVERNING THE FIELD}

We now return to the case of the field and show that an analogous assumption about the boundary contribution may not be made. Consider the wave equation

$$
\nabla^{2} \mathbf{B}-\frac{1}{c^{2}} \frac{\partial^{2} \mathbf{B}}{\partial t^{2}}=-\frac{4 \pi}{c} \nabla \times \mathbf{j}
$$

governing the magnetic field; Equation (12) may be obtained by simply taking the curl of the wave equation for the vector potential [Eq. (7) for $\mu=1,2,3]$. We write the solution to the initial-boundary value problem for Eq. (12), in analogy with Eq. (8), as

$$
\begin{aligned}
B_{k}\left(\mathbf{x}_{\mathrm{P}}, t_{\mathrm{P}}\right)= & \frac{1}{c} \int_{0}^{t_{\mathrm{P}}} \mathrm{d} t \int_{V} \mathrm{~d}^{3} x(\boldsymbol{\nabla} \times \mathbf{j})_{k} G+\frac{1}{4 \pi} \int_{0}^{t_{\mathrm{P}}} \mathrm{d} t \int_{\Sigma} \mathrm{d} \mathbf{S} \cdot\left(G \nabla B_{k}-B_{k} \nabla G\right) \\
& -\frac{1}{4 \pi c^{2}} \int_{V} \mathrm{~d}^{3} x\left(B_{k} \frac{\partial G}{\partial t}-G \frac{\partial B_{k}}{\partial t}\right)_{t=0},
\end{aligned}
$$

where $k=1,2,3$ designate the Cartesian components of $\mathbf{B}$ and $\boldsymbol{\nabla} \times \mathbf{j}$.

Here, we no longer have the freedom, offered by a gauge transformation in the case of Eq. (8), to make the boundary term zero, nor does this term always decay faster than the source term, so that it could be neglected for a boundary that tends to infinity, as is commonly assumed in textbooks (e.g., page 246 of Ref. [16]) and the published literature. ${ }^{25|26| 27|28| 29}$ The boundary contribution to the retarded solution of the wave equation governing the field [the second term on the right-hand side of Eq. (13)] entails a surface integral over the boundary values of both the field and its gradient. For the rotating superluminal source $(3)$, where the gradient of the field increases as $R_{\mathrm{P}}^{7 / 2}$ over a solid angle that decreases as $R_{\mathrm{P}}^{-4}$, this boundary contribution is proportional to $R_{\mathrm{P}}^{-1 / 2}$ (see Ref. [17]). Not only is this not negligible relative to the contribution from the source term [the first term on the right-hand side of Eq. [13]], but the boundary term constitutes the dominant contribution to the radiation field in this case.17

If one ignores the boundary term in the retarded solution of the wave equation governing the field, as in Refs. [16, 25, 26, 27, 28, 29], one obtains a different result, in the superluminal regime, from that obtained by calculating the field via the retarded potential. ${ }^{913132}$ This apparent contradiction stems solely from having ignored a term in the solution to the wave equation that is, by a factor of the order of $R_{\mathrm{P}}^{1 / 2}$, larger than the term that is normally kept. The contradiction disappears once we take the neglected term into account: the solutions to the wave equations governing both the potential and the field predict that the field of a 
rotating superluminal source decays as $R_{\mathrm{P}}^{-1 / 2}$ as $R_{\mathrm{P}}$ tends to infinity, a result that has been demonstrated experimentally $\underline{6] 7}$

We note, furthermore, that the representation

$$
\mathbf{A}\left(\mathbf{x}_{\mathrm{P}}, t_{\mathrm{P}}\right)=\frac{1}{c} \int \mathrm{d}^{3} x \frac{[\mathbf{j}(\mathbf{x}, t)]}{\left|\mathbf{x}-\mathbf{x}_{\mathrm{P}}\right|},
$$

of the retarded potential is differentiable as a classical (as opposed to generalized) function only in the case of a moving source whose speed does not exceed that of the waves it generates. The steps, familiar from the subluminal regime, one takes to derive Eq. (2) by differentiating Eq. (14) as a classical function are not mathematically permissible when the moving source has volume elements that approach the observer with the wave speed and zero acceleration at the retarded time. ${ }^{18 \mid 19}$ Contrary to the usual assumption, $\frac{25|26| 27|28| 29}{2}$ the retarded distribution of the density of a moving source is not necessarily smooth and differentiable if its rest-frame distribution is. The retarded distribution of a rotating source with a moderate superluminal speed is in general spread over three disjoint volumes (differing in shape from each other and from the volume occupied by the source in its rest frame) whose boundaries depend on the spacetime position of the observer. The limits of integration in Eq. (14), which delineate the boundaries of the retarded distribution of a localized source, are not differentiable functions of the coordinates of the observer at those points on the source boundary that approach the observer, along the radiation direction, with the speed of light at the retarded time. $\frac{1819}{1 n}$ the superluminal regime, derivatives of the integral representing the retarded potential are well-defined only as generalized functions. 8

\section{SUMMARY AND CONCLUSIONS}

We must stress that we have not calculated the radiation field directly from the solution to the wave equation governing the field (the solution whose boundary term is normally neglected) ${ }^{25 / 26 / 27 / 28 / 29}$ We have instead first solved the wave equation governing the potential (whose solution has no boundary term) and then used this solution to evaluate the neglected term in the exact version of the retarded solution for the field [Eq. (13)]. What one obtains by including the boundary term in the retarded solution to the wave equation governing the field is merely a mathematical identity; it is not a solution that could be used to calculate the field arising from a given source distribution in free space. Unless its boundary term happens 
to be small enough relative to its source term to be neglected, a condition that cannot be known a priori, the solution in question would require that one prescribe the field in the radiation zone (i.e., what one is seeking) as a boundary condition. Thus, the role of the classical expression for the retarded potential in radiation theory is much more fundamental than that of the corresponding retarded solution of the wave equation governing the field. The only way to calculate the free-space radiation field of an accelerated superluminal source is to calculate the retarded potential and differentiate the resulting expression to find the field ${ }^{9[17}$ (see also Refs. [31,32]).

A. A. is supported by the Royal Society. J. S., J. F., and A. S. are supported by U.S. Department of Energy grant LDRD 20080085DR, "Construction and use of superluminal emission technology demonstrators with applications in radar, astrophysics and secure communications."

1 Heaviside, O., Electrical Papers (Macmillan and Co., New York and London, 1894), 1st ed.

2 Sommerfeld, A., "Zur Elektronentheorie (3 Teile)", Nach. Kgl. Ges. Wiss. Göttingen Math. Naturwiss. Klasse 99-130, 363-439(1904); 201-235(1905).

3 Einstein, A., "Zur Elektrodynamik bewegter Körper", Annalen der Physik 17, 891-921 (1905).

4 Bolotovskii, B. M. and Ginzburg, V. L., "The Vavilov-Čerenkov effect and the Doppler effect in the motion of sources with superluminal velocity in vacuum", SOVPU 15, 184-192 (1972).

5 Ginzburg, V. L., "Vavilov-Čerenkov effect and anomalous Doppler effect in a medium in which wave phase velocity exceeds velocity of light in vacuum", SOVPJETP 35, 92-93 (1972).

6 Ardavan, A., Hayes, W., Singleton, J., Ardavan, H., Fopma, J., and Halliday, D., "Experimental observation of nonspherically-decaying radiation from a rotating superluminal source," J. Appl. Phys. 96, 7760-7777(E)(2004). Corrected version of 96, 4614-4631.

7 Singleton, J., Ardavan, A., Ardavan, H., Fopma, J., Halliday D. and Hayes, W., "Experimental demonstration of emission from a superluminal polarization current - a new class of solid-state source for MHz-THz and beyond", IEEE Digest 04EX857, 591-592 (2004).

8 Ardavan, H., Ardavan, A., Singleton, J., Fasel, J., and Schmidt, A., "Spectral and polarization characteristics of the nonspherically decaying radiation generated by polarization currents with 
superluminally rotating distribution patterns," J. Opt. Soc. Am. A 21, 858-872 (2004).

9 Ardavan, H., Ardavan, A., Singleton, J., Fasel, J., and Schmidt, A., "Morphology of the nonspherically decaying radiation field generated by a rotating superluminal source," J. Opt. Soc. Am. A 24, 2443-2456 (2007).

10 Schmidt, A., Ardavan, H., Fasel, J., Singleton, J., and Ardavan, A., "Occurrence of concurrent 'orthogonal' polarization modes in the Liénard-Wiechert field of a rotating superluminal source," in Becker, W. and Huang, H. H., eds., MPE Report 291, Proc. 363rd WE-Heraeus Seminar on Neutron Stars and Pulsars, pp. 124-127 (2007).

11 Bolotovskii, B. M. and Serov, A. V., "Radiation of superluminal sources in empty space", Phys. Usp. 48, 903-915 (2005).

12 Bessarab, A. V., Gorbunov, A. A., Martynenko, S. P., and Prudkoy, N. A., "Faster-than-light EMP source initiated by short X-ray pulse of laser plasma," IEEE Trans. Plasma Sci. 32, 1400-1403 (2004).

13 Ardavan, A. and Ardavan, H., "Apparatus for generating focused electromagnetic radiation," International patent application, PCT-GB99-02943 (September 6, 1999).

14 Ardavan, H., Ardavan, A., Singleton, J., and Perez, M. R., "Mechanism of generation of the emission bands in the dynamic spectrum of the Crab pulsar," Mon. Not. R. Astron. Soc. 388, 873-883 (2008).

15 Ardavan, A., Ardavan, H., Fasel, J., Middleditch, J., Perez, M. R., Schmidt, A., Singleton, J., "A new mechanism for generating pulsar-like polarization," in proceedings of Polarimetry days in Rome: Crab status, theory and prospects, Proceedings of Science (CRAB2008)016, http://pos.sissa.it (2009).

16 Jackson, J. D., Classical Electrodynamics (Wiley, New York, 1999), 3rd ed.

17 Ardavan, H., Ardavan, A., Singleton, J., Fasel, J., and Schmidt, A., "Fundamental role of the retarded potential in the electrodynamics of superluminal sources," J. Opt. Soc. Am A 25, 543-557 (2008).

18 Ardavan, H., "Reply to comments on 'Generation of focused, nonspherically decaying pulses of electromagnetic radiation'," Phys. Rev. E 62, 3010-3013 (2000).

19 Ardavan, H., "Method of handling the divergences in the radiation theory of sources that move faster than their waves," J. Math. Phys. 40, 4331-4336 (1999).

20 Conservation of energy is not violated by the subbeams; enhancement of the radiation fields 
within the subbeams is exactly compensated by reduction of the polar widths of the subbeams, so that the flux of energy across their cross sections remains the same at all distances from the source. The physical mechanism underlying the properties of the subbeams is the focusing in the time domain possible when a source both travels faster than its own waves and accelerates; contributions towards the intensity of the radiation made over an extended period of emission time are received during a significantly shorter period of observation time.

21 Ardavan, H., "Generation of focused, nonspherically decaying pulses of electromagnetic radiation," Phys. Rev. E 58, 6659-6684 (1998).

22 Ardavan, H., Ardavan, A., Singleton, J., "Frequency spectrum of focused broadband pulses of electromagnetic radiation generated by polarization currents with superluminally rotating distribution patterns," J. Opt. Soc. Am. A 20, 2137-2155 (2003).

23 Lyne, A.G., Graham-Smith F., Pulsar Astronomy (Cambridge U Press, Cambridge, 2006).

24 Słowikowska, A., Jessner, A., Klein, B., Kanbach, G., astro-ph/051 1599v2 (2005).

25 Hannay, J. H., "Bounds on fields from fast rotating sources, and others," Proc. R. Soc. London, Ser. A 452, 2351-2354 (1996).

26 Hannay, J. H., "Comment II on 'Generation of focused, nonspherically decaying pulses of electromagnetic radiation'," Phys. Rev. E 62, 3008-3009 (2000).

27 Hannay, J. H., "Comment on 'Method of handling the divergences in the radiation theory of sources that move faster than their waves'," J. Math. Phys. 42, 3973-3974 (2001).

28 Hannay, J. H., "Spectral and polarization characteristics of the nonspherically decaying radiation generated by polarization currents with superluminally rotating distribution patterns: comment," J. Opt. Soc. Am. A 23, 1530-1534 (2006).

29 Hannay, J. H., "Morphology of the nonspherically decaying radiation generated by a rotating superluminal source: comment,” J. Opt. Soc. Am. A 25, 2165-2166 (2008).

30 Morse, P. M. and Feshbach, H., Methods of Theoretical Physics (McGraw-Hill, New York, 1953), Pt. I.

31 Ardavan, H., Ardavan, A., Singleton, J., Fasel, J., and Schmidt, A., "Spectral and polarization characteristics of the nonspherically decaying radiation generated by polarization currents with superluminally rotating distribution patterns: reply to comment," J. Opt. Soc. Am. A 23, 1536-1539 (2006).

32 Ardavan, H., Ardavan, A., Singleton, J., Fasel, J., and Schmidt, A., "Morphology of the non- 
spherically decaying radiation field generated by a rotating superluminal source: reply to comment," J. Opt. Soc. Am. A 25, 2167-2169 (2008).

33 Singleton, J., Sengupta, P., Middleditch, J., Graves, T.L., Perez, M.R., Ardavan, H., and Ardavan, A., "Violation of the inverse square law by the observed flux-distance relationship for pulsars", Nature Magazine (London), submitted (2009).

34 Efstathiou, G., Ellis, R.S. and Peterson, B.A., "Analysis of a complete galaxy redshift surveyII the field-galaxy luminosity function", Mon. Not. R. Astr. Soc. 232, 431-461 (1988).

\section{APPENDIX A: A NOTE ON INTEGRATION BOUNDARIES}

It has been suggested ${ }^{29}$ that our demonstration of the nonspherical decay of the field of a rotating superluminal source can be dismissed on the grounds that the boundary term in Eq. (13) must be identically zero. However, the claim that the boundary contribution to the retarded solution of the wave equation governing the field is exactly zero even when the boundary encloses a source violates the very foundations of diffraction theory: For a volume $V$ in which no sources are present, Eq. 13 reduces to

$$
B_{k}\left(\mathbf{x}_{\mathrm{P}}, t_{\mathrm{P}}\right)=\frac{1}{4 \pi} \int_{0}^{t_{\mathrm{P}}} \mathrm{d} t \int_{\Sigma} \mathrm{d} \mathbf{S} \cdot\left(G \nabla B_{k}-B_{k} \nabla G\right),
$$

under the null initial conditions $\left.B_{k}\right|_{t=0}=\left(\partial B_{k} / \partial t\right)_{t=0}=0$. As in the customary geometry for diffraction, the closed surface $\Sigma$ can consist of two disjoint closed surfaces, $\Sigma_{\text {inner }}$ and $\Sigma_{\text {outer }}$ (e.g., two concentric spheres), both of which enclose the source (see Fig. 10.7 of Ref. [16]). If the observation point does not lie in the region between $\Sigma_{\text {inner }}$ and $\Sigma_{\text {outer }}$, i.e., lies outside the closed surface $\Sigma$, then the composite surface integral in Eq. (A1) vanishes:

$$
\begin{aligned}
\int_{0}^{t_{\mathrm{P}}} \mathrm{d} t \int_{\Sigma} \mathrm{d} \mathbf{S} \cdot\left(G \nabla B_{k}-B_{k} \nabla G\right) & =\int_{0}^{t_{\mathrm{P}}} \mathrm{d} t\left(\int_{\Sigma_{\text {inner }}}+\int_{\Sigma_{\text {outer }}}\right) \mathrm{d} \mathbf{S} \cdot\left(G \nabla B_{k}-B_{k} \nabla G\right) \\
& =0
\end{aligned}
$$

(see Ref. [30]). Under no circumstances, however, would the integrals over $\Sigma_{\text {inner }}$ or $\Sigma_{\text {outer }}$ vanish individually if these surfaces enclose a source, i.e., if there is a nonzero field inside $\Sigma_{\text {inner }}$; nor does the invariance of the values of these integrals under deformations of $\Sigma_{\text {inner }}$ and $\Sigma_{\text {outer }}$ have any bearing on whether they are nonvanishing or not. ${ }^{29}$ Equation (A1) forms the basis of diffraction theory! $\frac{16}{16}$ the surface integrals over $\Sigma_{\text {inner }}$ and $\Sigma_{\text {outer }}$ were to 
vanish individually, as claimed in Ref. [29], the diffraction of electromagnetic waves through apertures on a surface enclosing a source would be impossible (see Sec. 10.5 of Ref. [16]). 